\title{
Phase Space Framework for Coherence: Towards Quantum Computing System Design
}

\author{
T. Ganesan* \\ Generation/Fuels \& Combustion, TNB Research No. 1, Lorong Ayer Itam, \\ Kawasan Institusi Penyelidikan,43000 Kajang, Selangor Darul Ehsan, Malaysia \\ *tim.ganesan@gmail.com
}

\begin{abstract}
This work aims to propose a framework for the phase space representation of quantum coherence in matter. The central thesis of this work is that quantum coherence could result from weak time-periodic forcing in the absence of other types of interactions (or measurements) from the environment. Therefore the Floquet formalism is employed to depict the coherent quantum system. A phase space representation for coherent quantum systems is formulated and the resulting three uncertainty principles are presented. The mathematical structure for quantum coherent transport is constructed via a non-equilibrium thermodynamic approach. The framework presented in this paper is targeted towards the development of nanosystems and other elements; employed to design systems for quantum computing.
\end{abstract}

Keywords: quantum coherence; phase space; Floquet formalism; uncertainty principles; quantum coherent transport; quantum computing

\section{Background}

Critical quantum mechanical phenomena in quantum computing are; entanglement, superposition and tunneling. These phenomena are closely linked to quantum coherence. Quantum systems exist in a coherent state prior to collapsing into a single quantum state. This process of collapse called decoherence usually occurs when the quantum state interacts with the environment and loses energy or undergoes measurement. Thus in a coherence state, the quantum system loses its discreet properties and becomes continuous similar to a classical system. Some interesting examples of quantum coherence in matter are superfluidity or Bose-Einstein Condensate [1] and superconductivity [2]. In quantum optics, Sudarshan (1963) [3] and Glauber (1963) [4] laid the foundations of the quantum theory of coherent light and their P-representation provided the phase space formulation of such systems. These theories led to a significant development in laser science and the physics of non-classical light [5].

Explanations on biological processes often revolve around ideas from quantum mechanics [5], [6]. For instance: explanations on the olfactory mechanism [7] dynamics of photosynthetic transport in plant [8] and theories on avian magnetoreception [9]. In these processes, ideas involving quantum entanglement, superposition and tunnelling are often employed. In addition, to provide a solid theoretical framework for energy transfer mechanisms in biological systems, quantum analogues of thermodynamical systems such as heat engines have been developed. In the quantum version of heat engines, instead of steam and heat coherent electrons and photons are utilized [10]. Thus, quantum coherence has been employed conceptually to explain many observations in physics as well as biology. However, the mathematical foundations of quantum coherence in matter remain vastly unexplored.

The main aim is to propose a theoretical framework for the phase space representation of quantum coherence in matter. The primary hypothesis used in this work is the idea that quantum coherence results from weak time-periodic forcing in the absence of other types of interactions from the environment. Therefore it is possible to represent a coherent quantum system in terms of Floquet formalism [11]. Subsequent theoretical arguments and constructs based on this idea are presented in this paper.

This paper is organized as follows: Section 2 and Section 3 provide details on the Floquet formalism for coherent states in quantum systems. Section 4 introduces the phase space formulation of coherent quantum states while Section 5 presents details regarding the coherent phase space transform. The uncertainty principles which results from the mentioned phase space formulation are given in Section 6. Section 7 discusses the dynamical aspects of the coherent transport of matter within a non-equilibrium framework. Finally, this paper ends with some concluding remarks and suggestions for future works.

\section{Floquet Formalism \& Coherent States}

Quantum coherent states can be thought of specific states of the quantum harmonic oscillator. In these states, the harmonic oscillator loses its discreet quantum properties and adopts the behaviour of a classical 
harmonic oscillator. Therefore quantum systems in coherent states have minimum uncertainty which could be shown by proving the squeezed uncertainty principle. A coherent quantum state, $|\alpha\rangle$ is defined as follows:

$a(t)|\alpha\rangle=e^{-i \omega t} a(0)|\alpha\rangle \quad$ where $\quad \alpha \in C$

where $\mathrm{w}$ is the angular momentum. In the Schrödinger picture the coherent state, $|\alpha\rangle$ can be described as:

$\alpha(t)=e^{-i \omega t} \alpha(0)$

such that $a|\alpha(t)\rangle=\alpha(t)|\alpha(t)\rangle$ resulting in $a=\alpha(t)$. In this approach the Schrödinger equation becomes:

$H(t) \psi^{\alpha}(x, t)=\sqrt{\frac{m \omega}{2 \hbar}}\left(x+\frac{\hbar}{m \omega} \frac{\partial}{\partial x}\right) \psi^{\alpha}(x, t)=\alpha(t) \psi^{\alpha}(x, t)$

where $\psi^{\alpha}(t)$ is the wave function of coherent state $|\alpha\rangle, H(t)$ is the Hamiltonian, $m$ is the particle mass and $\hbar$ is the Planck's constant. A similar approach could be carried out using the Floquet formulation. Let $|\phi(t)\rangle$ be the Floquet wave function. Then the Schrodinger wave function, $|\psi(t)\rangle$ could be transformed from the $|\phi(t)\rangle$ as follows:

$|\psi(t)\rangle=e^{-i \eta^{\prime} t / \hbar}|\phi(t)\rangle$

where $\eta^{\prime}$ is the Floquet quasienergy. Here analogues from equation (2) are drawn by setting $\eta^{\prime}=\omega$ and $\phi(t)=\psi(0) \quad$ resulting $\quad$ in $\quad|\phi(t)\rangle=e^{i \eta^{\prime} t}|\psi(t)\rangle . \quad$ Setting $\quad t \quad=\quad 0$, this equation becomes $|\phi(0)\rangle=e^{i \eta^{\prime} t}|\psi(0)\rangle=e^{i \eta^{\prime} t}|\phi(t)\rangle$. Note that $|\phi(t)\rangle=e^{-i \eta^{\prime} t}|\phi(0)\rangle$ is similar to the quantum coherent transformation in equation (2). Similarly this would result in the following Schrodinger equation:

$H(t) \phi(x, t)=\eta \phi(x, t)$

Therefore quantum coherent states could be accurately described using the Floquet wave function, $\phi(x, t)$. It is important to note that when the Floquet formalism is employed in the coherent case, the $\eta$ in equation (5) is not the Floquet quasienergy $\left(\eta^{\prime}\right)$ but another form of coherent quasienergy (which arises similarly as the Floquet quasienergy arises when the Floquet transformation is carried out).

\section{Floquet-Type Schrödinger Equation}

The framework presented in this work is built from the premise that quantum coherence could result from weak time-periodic forcing in the absence of other interactions or measurements. Initially Schrodinger equation is defined as follows:

$i \hbar \frac{\partial \psi(x, t)}{\partial t}=\left(-\frac{\hbar^{2}}{2 m} \frac{\partial^{2}}{\partial x^{2}}+V(x, t)\right) \psi(x, t)$

where the time-periodic potential is defined as follows:

$V(x, t)=V_{o}+V_{1} \cos (\omega t) \quad$ where $\quad a \leq x \leq b$

According to the Floquet theorem, the wave function from a time-periodic system has the form of equation (4) where $\phi(t)$ is periodic such that $\phi(t)=\phi(t+T)$ with the period $T=2 \pi / \omega$ with $\eta^{\prime}$ is its Floquet quasienergy. As mentioned in the previous section, in this work $\eta^{\prime}$ is replaced by $\eta$ which is the coherent quasienergy. The energy of the system, that $E^{\prime}$ is usually defined as by $E^{\prime}=\eta^{\prime}+n \hbar \omega$ where $n$ can be defined as the $n$-th side-band energy. Since in this work the energy of the system describes coherent states, $E$ is defined in as its minimal side-band energy, $n=1$ :

$E=\eta+\hbar \omega$

Performing the Floquet transform (equation (4)), the resultant Floquet-type Schrodinger equation employed to describe coherent states is as follows:

$-i \hbar \frac{\partial \phi(x, t)}{\partial t}-\frac{\hbar^{2}}{2 m} \frac{\partial^{2} \phi(x, t)}{\partial x^{2}}+\left[V_{o}+V_{1} \cos (\omega t)\right] \phi(x, t)=\eta \phi(x, t)$ 
Via separation of variables, Equation (9) is split into the following differential equations:

$$
\begin{aligned}
& -\frac{\hbar^{2}}{2 m} \frac{d^{2} g(x)}{d x^{2}}+V_{o} g(x)=E g(x) \\
& i \hbar \frac{d f(t)}{d t}-V_{1} \cos (\omega t) f(t)=(E-\eta) f(t)
\end{aligned}
$$

These differential equations yield the following solution for the given potential boundaries:

$$
\left.\phi(x, t)=\exp [-i(E-\eta) t / \hbar] \llbracket a_{o} e^{q x}+b_{o} e^{-q x}\right] \text { where } q=\sqrt{2 m\left(V_{o}-\eta-\hbar \omega\right) / \hbar^{2}}
$$

where $a_{o}$ and $b_{o}$ are constants. Therefore using this approach, the obtained Floquet wave function $\phi(x, t)$ is used to describe the coherent state of a quantum system.

\section{Phase Space Formulation For Coherent Quantum Systems}

In the phase space formulation of quantum physics introduced in Wigner (1932) [12], the Fourier transform is employed to transform the position operator, $\hat{x}$ into the momentum operator, $\hat{p}$. Therefore quantum density functions, $\psi(x, t)$ could be represented in a classical setting or phase space as $\varphi(p, t)$ with the aid of the Wigner transform. Although this is possible with standard quantum systems, to the best of the author's knowledge classical representations for coherent quantum systems are not well explored. Such a classical representation or a phase space formulation for coherent quantum systems is what this work aims to establish. As mentioned previously, the momentum operator could be obtained by the complete Fourier transform of the position operator. Similarly the relation of energy to momentum and position is considered. Energy could be thought of as the rotation of momentum and space in a quantum setting. It is known that $E=\hbar \omega$ and $\mathbf{p}=\hbar \mathbf{k}$ with $\mathbf{k}$ as the wave number (vector). Considering $\mathbf{k}$ to be one-dimensional the vector notation could be omitted giving $p=\hbar k$. Since the phase velocity, $v_{p}=\omega / k=E / p$ therefore:

$$
E=p v_{p}=\frac{p \omega}{k}=\omega\left(\frac{p}{k}\right)
$$

If we define the rotation, $R_{\theta}=\omega t$, then the energy, $E$ could be formulated as follows:

$$
E=R_{\theta}\left(\frac{p}{k t}\right)
$$

Therefore energy could be thought of as the rotation of momentum, $p$ or space (wave number), $k$. Since the phase space representation of collapsed quantum systems is a complete Fourier transform, it is reasonable to conjecture that coherent (or non-collapsed) quantum systems could be represented by some form of partial Fourier transform such as the Fractional Fourier Transform [13]. The Fractional Fourier Transform, $F_{\theta}$ of a function $f(t)$ is given as follows:

$F_{\theta}: f(t) \rightarrow F(u)$

$$
\begin{aligned}
& F_{\theta}(f(t))=F(u)=\int_{-\infty}^{+\infty} f(t) K_{\theta}(u, t) d t \\
& K_{\theta}(u, t)=\left\{\begin{array}{clc}
\sqrt{\frac{1-i \cot \theta}{2 \pi}} \exp \left(j \frac{t^{2}+u^{2}}{2} \cot \theta-i u t \cdot \csc \theta\right) & \text { if } & \theta \neq n \pi \\
\delta(t-u) & \text { if } & \theta=2 n \pi \\
\delta(t+u) & \text { if } & \theta=(2 n \pm 1) \pi
\end{array}\right.
\end{aligned}
$$

where $n \in N$ and $\delta(\cdot)$ is the Dirac delta function. Since the Fractional Fourier Transform is a rotated Fourier transform, thus the resulting phase space would not be momentum-space but rather an energy-space. This is because as mentioned previously, energy can be defined as the rotation of space or momentum. 


\section{Coherent Phase Space Transform}

In this section the formulation of the proposed phase space transformations for coherent quantum systems is discussed. The Fractional Fourier Transform is utilized to transform the coherent quantum system to the coherent phase space:

$$
F_{\theta}: \phi(x, t) \rightarrow \varphi(\varepsilon, t)
$$

where $\phi(x, t)$ is the Floquet wave function used to represent the coherent quantum system, $\varphi(\varepsilon, t)$ is its phase space counterpart where $\varepsilon$ is the coherent energy. As mentioned in the previous section, the fractional Fourier Transform maps the coherent quantum wave function to its energy space. The coherent phase space representation of equation (12) is shown in this section. Firstly, the Floquet wave function, $\phi(x, t)$ decomposed into its time and spatial components $\phi(x, t)=f(t) g(x)$ where:

$$
f(t)=\exp [-i(E-\eta) t / \hbar]
$$

and setting the constants $a_{o}=b_{o}$,

$$
g(x)=a_{o}\left(e^{q x}+e^{-q x}\right)=a_{o} \cosh (q x)
$$

Therefore the transformation for $\theta \in(0,1)$ would yield:

$$
\begin{aligned}
\varphi(\varepsilon, t) & =F_{\theta}\{\phi(x, t)\} \\
& =F_{\theta}\{f(t) g(x)\} \\
& =f(t) F_{\theta}\{g(x)\} \\
\varphi(\varepsilon, t)= & f(t) a_{o} \sqrt{1+i \tan \theta} \exp \left(-\frac{i}{2} \tan \theta\left(\varepsilon^{2}-q^{2}\right)\right) \cos (\varepsilon q i \sec \theta)
\end{aligned}
$$

It should be noted that the phase space trajectory $\varphi(\varepsilon, t)$ is in complex form. If $\theta=1$ is fixed, then the transformation becomes the conventional Fourier transform where the Floquet wave function is transformed to its counterpart in momentum-space. Conversely, by setting $\theta=0$, no transformation takes place and the Floquet wave function remains the same.

It was previously established that the Floquet wave function employed here depicts a quantum system in coherent form. Hence the parameter $\theta$ gives a measure on the degree at which the coherence affects its classical trajectory. For instance, if the coherence effects are minimal classically then $\theta \rightarrow 1$ and the coherent wave function would be transformed to momentum-space. On the contrary, if the level of coherence highly influences its classical trajectory, then $\theta \in(0,1)$ (or in the extreme case, $\theta \rightarrow 0$ ) where the coherent wave function would be transformed to energy-space. This brings up the interesting notion that the coherence in quantum systems affects their classical representations on various degrees (depending on $\theta$ ).

In the Wigner's phase space formulation, the construction of phase space probability distributions were plagued by problems arising from Heisenberg's Uncertainty Principle. This is because the Uncertainty Principle poses various restrictions on the simultaneous measurements of position and momentum. To resolve this issue, Wigner introduced the concept of pseudo-probabilities giving rise to the Wigner distribution [14]:

$$
P(x, p, t)=\frac{1}{\pi \hbar} \int_{-\infty}^{+\infty} \psi^{*}(x+y, t) \psi(x-y, t) e^{2 i p y / \hbar} d y
$$

where $\psi(x, t)$ is the wave function and $\psi^{*}(x, t)$ its corresponding conjugate. In this work, such pseudoprobabilities are constructed for the coherent phase space using a fractional framework. It is well known that the Wigner Transform originates from the Fourier Transform. Similarly the Fractional Wigner Transform corresponds to the Fractional Fourier Transform. The Fractional Wigner Transform could be obtained by performing a rotation operation on the function (i.e. Wigner Distribution) obtained from the conventional Wigner Transform [15]:

$$
\begin{aligned}
& R_{\theta}: W(x, y) \rightarrow W_{\theta}\left(x^{\prime}, y^{\prime}\right) \\
& W_{\theta}\left(x^{\prime}, y^{\prime}\right)=W(x \cos \theta-y \sin \theta, x \sin \theta+y \cos \theta)
\end{aligned}
$$

where $W_{\theta}(x, y)$ is the Fractional Wigner function and. $R_{\theta}$ is the rotation operator. The remaining of this section consists of the construction of the pseudo-probability distribution for coherent quantum systems via the 
Fractional Wigner Transform. The conventional Wigner Transform is applied to the coherent wave function, $\phi(x, t)$ :

$W(x, \varepsilon)=\int_{-\infty}^{+\infty} \phi\left(\frac{x+y}{2}\right) \phi^{*}\left(\frac{x-y}{2}\right) \exp (-2 \pi i \varepsilon y) d y$

After some mathematical derivations (Appendix A), the following relation is obtained:

$W(x, \varepsilon)=a_{o}^{2} \delta\left(\varepsilon^{2}-\frac{m \omega}{\pi^{2} \hbar}\right)$

The Fractional Wigner Transform is then carried out by applying the rotation operator to the Wigner distribution in equation (24). By substituting $x=x \cos \theta-\varepsilon \sin \theta$ and $\varepsilon=x \sin \theta+\varepsilon \cos \theta$, the following distribution is obtained:

$W_{\theta}(x, \varepsilon)=a_{o}^{2} \delta\left((x \sin \theta+\varepsilon \cos \theta)^{2}-\frac{m \omega}{\pi \hbar}\right)$

The form of the Wigner function obtained in equation (25) is not very useful since it takes only infinite or zero values. Therefore we depict equation (25) in a series representation given as follows:

$$
\begin{aligned}
W_{\theta}(x, \varepsilon) & =a_{o}^{2} \delta\left((x \sin \theta+\varepsilon \cos \theta)^{2}-\frac{m \omega}{\pi \hbar}\right) \\
& =a_{o}^{2} \sum_{k=-\infty}^{\infty} \exp \left(i k\left((x \sin \theta+\varepsilon \cos \theta)^{2}-i k \frac{m \omega}{\pi \hbar}\right)\right.
\end{aligned}
$$

Taking $\Theta=(x \sin \theta+\varepsilon \cos \theta)^{2}-\frac{m \omega}{\pi \hbar}$, the series is expanded as follows:

$$
\begin{aligned}
W_{\theta}(x, \varepsilon)= & a_{o}^{2} \sum_{k=-\infty}^{\infty} \exp (i k \Theta) \\
& =a_{o}^{2} \sum_{k=-\infty}^{\infty}[\cos (k \Theta)+i \sin (k \Theta)]
\end{aligned}
$$

This way the Wigner function, $W_{\theta}(x, \varepsilon)$ is split into its real and complex components. It should be noted that the Wigner function must be real throughout. Thus, the complex part of the Wigner function is neglected resulting in:

$W_{\theta}(x, \varepsilon)=a_{o}^{2} \sum_{k=-\infty}^{\infty} \cos (k \Theta)$

When the Wigner function takes negative real values, then it can be interpreted as the level of interference or coherence of the quantum system. As can be seen in equations (20) and (27), in this work the Wigner function takes on complex values. Although the complex part of equation (27) is neglected for the sake of practicality, nevertheless its physical significance is worthy of further consideration. Besides, there is currently no physical explanation for a complex-valued Wigner function in phase space. One may conjecture that the complex-valued Wigner function may be the manifestation of macroscopic coherence (or macroscopic non-classical states) as seen in some experiments [16].

\section{Uncertainty Principles}

In Fourier analysis, the principle that a signal cannot be localized simultaneously in time and frequency gives rise to the uncertainty principle. Similarly the Heisenberg's Uncertainty Principle could be arrived upon as a direct result of the phase space Fourier Transform relating position and momentum [17]. As with the conventional Fourier Transform, uncertainty principles also arise in effect of the Fractional Fourier Transform [18].

The Fractional Fourier Transform was employed in this work to formulate the phase space transform for coherent quantum systems. Hence it is inevitable for uncertainty principles involving position, $x$ and coherent energy, $\varepsilon$ to emerge from these coherent quantum systems. In Guan-Lei et al., (2010) [18], rigorous derivation of the uncertainty principles were presented. In that same work, it was shown that unlike the conventional Fourier Transform, the Fractional Fourier Transform produces three uncertainty relations. 
Uncertainty in the conventional Fourier sense refers to the concept of statistical variance of the related variables. This sort of uncertainty is portrayed in Heisenberg's Uncertainty Principle:

$$
\sigma^{2}(x) \sigma^{2}(p) \geq \frac{\hbar}{2}
$$

where the variance $\sigma^{2}(\cdot)$ represents the uncertainty in the physical variables position, $x$ and momentum, $p$. The application of the Fractional Fourier Transform causes the emergence of two types of uncertainties: variance, $\sigma^{2}(\cdot)$ and distortion (group-delay), $\tau(\cdot)$ :

$$
\begin{aligned}
& \sigma^{2}(x)=\int_{-\infty}^{+\infty} x^{2}\left|f_{\theta}(x)\right|^{2} d x \\
& \tau(x)=\int_{-\infty}^{+\infty}\left|\frac{f_{\theta}(x)}{d x}\right|^{2} d x
\end{aligned}
$$

where $f_{\theta}(x)$ is the function resulting from the Fractional Fourier Transform of $x$ with the angle, $\theta$. In the inequalities presented in this section, the term, $V$ and $\operatorname{sgn}(V)$ are defined as follows:

$$
V=\frac{\sin (2 \theta) \sin (2 \beta)}{4} \quad \text { and } \quad \operatorname{sgn}(V)=\left\{\begin{array}{ccc}
1 & \text { if } \quad V \geq 0 \\
-1 & \text { if } \quad V<0
\end{array}\right.
$$

Provided the variances are defined:

$$
\sigma^{2}(\varepsilon)=\int_{-\infty}^{+\infty} \varepsilon^{2}\left|f_{\beta}(\varepsilon)\right|^{2} d \varepsilon \quad \text { and } \quad \sigma^{2}(x)=\int_{-\infty}^{+\infty} x^{2}\left|f_{\theta}(x)\right|^{2} d x
$$

Then, the first inequality (variance-variance) is given as follows:

$$
\sigma^{2}(\varepsilon) \sigma^{2}(x) \geq \frac{(\cos \theta \sin \beta+\operatorname{sgn}(V) \cos \beta \sin \theta)^{2}}{4}
$$

where $\theta$ and $\beta$ are the Fractional Fourier Transform angles. Since in the coherent phase space transform only has a single angle and its transforms $x \rightarrow \varepsilon, f_{\beta}(\varepsilon)$ does not exist. This could be imposed by setting $\beta=0$ to arrive at the following uncertainty relation:

$$
\sigma^{2}(\varepsilon) \sigma^{2}(x) \geq \frac{(\sin \theta)^{2}}{4}
$$

Given that the distortions are defined as the following:

$$
\tau(\varepsilon)=\int_{-\infty}^{+\infty}\left|\frac{d f_{\beta}(\varepsilon)}{d \varepsilon}\right|^{2} d \varepsilon \quad \text { and } \quad \tau(x)=\int_{-\infty}^{+\infty}\left|\frac{d f_{\theta}(x)}{d x}\right|^{2} d x
$$

The second resulting uncertainty (distortion-distortion) is as the following:

$$
\tau(\varepsilon) \tau(x) \geq \frac{(\sin \theta \cos \beta+\operatorname{sgn}(V) \cos \theta \sin \beta)^{2}}{4}
$$

Setting the angle $\beta=0$ yields the following uncertainty principle:

$\tau(\varepsilon) \tau(x) \geq \frac{(\sin \theta)^{2}}{4}$

The variance and distortion are defined as follows:

$$
\sigma^{2}(x)=\int_{-\infty}^{+\infty} x^{2}\left|f_{\theta}(x)\right|^{2} d x \quad \text { and } \quad \tau(\varepsilon)=\int_{-\infty}^{+\infty}\left|\frac{d f_{\beta}(\varepsilon)}{d \varepsilon}\right|^{2} d \varepsilon
$$

The third inequality (variance-distortion) resulting from the Fractional Fourier Transform is given below:

$$
\sigma^{2}(x) \tau(\varepsilon) \geq \frac{(\cos \theta \cos \beta+\operatorname{sgn}(V) \sin \theta \sin \beta)^{2}}{4}
$$

Setting the angle $\beta=0$ results in: 


$$
\sigma^{2}(x) \tau(\varepsilon) \geq \frac{(\cos \theta)^{2}}{4}
$$

Interchanging the variable definitions for variance and distortion produces the same results:

$$
\sigma^{2}(x) \tau(\varepsilon)=\sigma^{2}(\varepsilon) \tau(x) \geq \frac{(\cos \theta)^{2}}{4}
$$

Details on the derivations of the inequalities (31), (34) and (37) resulting from the Fractional Fourier Transform is provided in Guan-Lei et al., (2010) [18]. Therefore the three main uncertainty principles obtained are:

Variance-Variance: $\sigma^{2}(\varepsilon) \sigma^{2}(x) \geq \frac{(\sin \theta)^{2}}{4}$

Distortion-Distortion: $\tau(\varepsilon) \tau(x) \geq \frac{(\sin \theta)^{2}}{4}$

Variance-Distortion: $\sigma^{2}(x) \tau(\varepsilon)=\sigma^{2}(\varepsilon) \tau(x) \geq \frac{(\cos \theta)^{2}}{4}$

\section{Non-Equilibrium Thermodynamics \& Coherent Transport}

Quantum systems in coherent states are very delicate and highly sensitive to interactions (or measurements) with the environment. Any such interactions would annihilate coherence causing the quantum state to collapse. Therefore transport phenomena of quantum systems in coherent states are very unlikely and difficult to achieve without the system undergoing quantum collapse. One theory that proposes a systematic mechanism for this phenomenon is 'noise-assisted transport' [19], [20]. In noise-assisted transport, a coherent quantum system is exposed with certain levels of noise sufficient enough to cancel-off the influence of the environment and sustain coherence of the quantum system. If the quantum system is exposed to high levels of noise, the noise would then act similar to the environment and cause the quantum system to collapse. On the other hand, if the amount of noise introduced is too low, then there would be no transport although the coherence of the quantum system is sustained (assuming the system is devoid of interactions from any other external environments). Thus the idea of noise-assisted transport is a very attractive and consistent theory that explains quantum coherent transport.

In this section, the mathematical description of noise-assisted transport is presented within the framework of the coherent phase space formulation. Quantum systems in coherent states are not in dynamical or mechanical equilibrium. It can be stated that these systems go into equilibrium when they undergo quantum collapse. Therefore in this work, a non-equilibrium thermodynamical approach is employed to describe coherent transport. The closeness between quantum coherence and classical systems enables the utilization of a thermodynamical approach with minimal glitches. In addition, the coherent phase space represents classical dynamics at which thermodynamical constructs (e.g. work and force) should hold.

This approach follows closely the Bochkov-Kuzovlev framework [21] as described in Campisi et al., (2011) [22]. It should be noted that unlike those research works, the non-equilibrium approach in this work does not attempt to formulate or prove quantum fluctuation relations. In Campisi et al., (2011) [22], the issue that work is not a quantum observable is raised. This argument does not surface since the work defined here is purely random classical work. This work is applied to the coherent phase space for transport (noise-assisted transport).

In coherent phase space, the coherent energy, $\varepsilon$ is seen similar to the Hamiltonian of the system. Due to coherence, the quantum system does not have a fixed position but rather randomly distributed positions.

Consider a non-equilibrium system undergoing quantum walk between two random points $x_{o}$ and $x_{1}$ in the coherent phase space:

$\Gamma\left(x_{o} ; \lambda\right):\left(\varepsilon_{o}, x_{o} ; t_{o}\right) \rightarrow\left(\varepsilon_{1}, x_{1} ; t_{1}\right)$

where $\Gamma\left(x_{o} ; \lambda\right)$ is the flow dependent on the initial condition and external force, $\lambda$. The system's quantum walk depends on the introduction of external random work, $w_{o}$ defined as follows:

$$
\begin{aligned}
w_{o}\left[x_{o} ; \lambda\right] & =\int_{x_{o}}^{x_{1}} \lambda d x \\
& =\varepsilon\left(\Gamma\left(x_{o} ; \lambda\right)\right)-\varepsilon\left(x_{o}\right)
\end{aligned}
$$


Following the derivations presented in Campisi et al., (2011) [22] by assuming microreversibity the following inequality for work is obtained:

$$
\left\langle w_{o}\right\rangle_{\lambda} \geq 0
$$

This means that on average the driven system would only absorb energy if it is perturbed out of equilibrium. This could be viewed as a version of the second law of thermodynamics. It is known that microreversibity is a property that is common to non-driven systems described by a time-dependent Hamiltonian [23]. The Floquet formulation is employed in this work to obtain the coherent wave function, $\phi(x, t)$. Therefore reversibility exists albeit in a periodic fashion due to the weak-driving with period, $T$ :

$$
\phi(x, t)=\phi(x, t+T)
$$

Rigorous derivations using the microreversibility principle is not carried out in this work since its only significance is in equation (45). Besides, as mentioned previously this framework does not involve fluctuation relations where the microreversibility principles would be indispensable. The probability of work when a force, $\lambda$ is applied to a coherent system could then be expressed as:

$$
P\left[x_{o} ; \lambda\right]=\int_{x_{1}}^{x_{2}} W_{\theta}\left(x_{o}, \varepsilon_{o}\right) \delta\left(w_{o}-\varepsilon\left(x_{1} ; t_{1}\right)-\varepsilon\left(x_{o} ; t_{o}\right)\right) d x_{o}
$$

where $x_{o}$ is randomly sampled from the pseudo-probability distribution, $W_{\theta}$ obtained in equation (28).

\section{Conclusions \& Future Research Directions}

Quantum coherence is the core of quantum computing. The formulations presented in this work aim to construct foundations to enable the manipulation and preservation of states in quantum computing devices. Such manipulation is only possible if we understand the classical effects and dynamics of quantum coherence. The fractional phase space transform and the uncertainty principles presented in this work does exactly this. Equipped with the pseudo-probabilities the mathematical framework to describe noise-assisted transport is shown within the non-equilibrium thermodynamic framework.

The Floquet formalism presented was employed to represent coherent quantum systems which result from weak periodic driving. It was shown that consequently these systems could be studied in a phase space setting via the utilization of Fractional Fourier Transforms. The phase space transform then gives rise to three uncertainty principles uncovering a new form of uncertainty: distortion (or group delay). In addition, the Fractional Wigner Transform could be used to obtain the pseudo-probabilities in phase space. Equipped with the pseudo-probabilities the mathematical framework to describe noise-assisted transport is shown within the nonequilibrium thermodynamic framework.

Investigations into conditions influencing the parameter $\theta$ could shed some light on the quantum coherence-classical mechanisms. This could be very useful for minimizing or maximizing classical effects produced by coherent quantum systems. Another interesting avenue of research is the complex form of the phase space trajectory and pseudo-probability obtained in equations (20) and (27). The physical conditions and implications of these complex forms could be investigated further. The conjecture that these complex forms indicate the presence of macroscopic quantum coherence should be verified. In addition, further research efforts could be directed towards a consistent incorporation of microreversibility into the proposed framework.

Noise-assisted transport provides a strong theoretical framework for describing quantum coherent transport. Nevertheless this framework and the formulations presented in this work do not account for time albeit it plays a critical role in the process of quantum coherence. Thus findings of Wheeler's delayed-choice experiments (retrocausal phenomenon) [24], [25], [26] should not be discounted when exploring quantum coherence. Further theoretical and empirical research into this aspect of quantum coherence would be very useful for increasing our understanding of this phenomenon. Further experimental works involving the classical representation of quantum coherence phenomena would be required to validate the mathematical framework presented in this work. A more complete understanding of quantum coherence in matter would lead to more efficient engineering of quantum systems - especially in fields such as nano and quantum-computing.

\section{Acknowledgments}

The author sincerely thanks the School of Chemical Engineering at the University of Adelaide, Australia and Tenaga Nasional Berhad - Research (TNBR), Malaysia for extending their resources for this research project.

\section{References}

[1]. Zwierlein, M.W., Stan, C. A., Schunck, C. H., Raupach, S. M. F., Gupta, S., Hadzibabic, Z., and Ketterle, W., Observation of Bose-Einstein Condensation of Molecules, Physical Review Letters, 91, 250401, (2003).

[2]. Cooper, L.N. and Feldman, D., BCS: 50 Years (book). World Scientific. ISBN 978-981-4304-64-1, (2010). 
[3]. Sudarshan, E.C.G., Equivalence of Semiclassical and Quantum Mechanical Descriptions of Statistical Light Beams, Physical Review Letters, 10, 277, (1963).

[4]. Glauber, R.J., Coherent and Incoherent States of the Radiation Field, Physical Review Letters, 131, 2766, (1963).

[5]. Gilchrist, A., Gardiner, C.W. and Drummond, P.D., Positive P representation: Application and validity, Physical Review A, 55, 3014, (1997).

[6]. McFadden, J., and Al-Khalili, J., A quantum mechanical model of adaptive mutation, Biosystems, 50, 203, (1999).

[7]. Gane, S., Georganakis, D., Maniati, K., Vamvakias, M., Ragoussis, N., Skoulakis, E.M. and Turin, L., Molecular vibration-sensing component in human olfaction. PloS one, 8, 55780, (2013).

[8]. Ghosh, P. K., Smirnov, A. Y. \& Nori, F., Quantum effects in energy and charge transfer in an artificial photosynthetic complex. Journal of Chemical Physics, 134, 244103, (2011).

[9]. Bandyopadhyay, J. N., Paterek, T. \& Kaszlikowski, D., Quantum coherence and sensitivity of avian magnetoreception. Physical Review Letters, 109, 110502, (2012).

[10]. Altintas, F., Hardal, A.Ü.C. and Müstecaplıoglu, Ö.E., Rabi model as a quantum coherent heat engine: From quantum biology to superconducting circuits, Physical Review A, 91, 023816, (2015).

[11]. Lee, C.C. and Ho, C.L., Quantum metastability in time-periodic potentials, Annals Phys., 320, 175, (2005)

[12]. Wigner, E., On the Quantum Correction for Thermodynamic Equilibrium, Physics Review, 40, 749, (1932)

[13]. Almeida, L.B., The Fractional Fourier Transform and Time-Frequency Representations, IEEE Transactions on Signal Processing, 42, 3084, (1994).

[14]. Belloni, M., Doncheski, M.A. and Robinett, R.W., Wigner quasi-probability distribution for the infinite square well: Energy eigenstates and time-dependent wave packets, American Journal of Physics, 72, 1183, (2004).

[15]. Lin, P.Y., The Fractional Fourier Transform and Its Applications, National Taiwan University, Taipei, Taiwan, ROC, (1999).

[16]. Lee, K.C., Sussman, B.J., Sprague, M.R., Michelberger, P., Reim, K.F., $\quad$ Nunn,J., Langford, N. K., Bustard, P.J., Jaksch, D. and Walmsley, I.A., Macroscopic non-classical states and terahertz quantum processing in room-temperature diamond, Nature Photonics, 6, 41, (2012).

[17]. Begué, M., 'The Uncertainty Principle: A Brief Survey', University of Maryland, (2012).

[18]. Guan-Lei, X., Xiao-Tong, W., and Xiao-Gang, X., Novel uncertainty relations associated with fractional Fourier transform. Chinese Physics B, 19, 014203, (2010).

[19]. Chin, A. W. and Huelga, S. F. and Plenio, M. B., Coherence and decoherence in biological systems: principles of noise-assisted transport and the origin of long-lived coherences, Philosophical Transactions of the Royal Society of London A: Mathematical, Physical and Engineering Sciences, 370, 3638, (2012).

[20]. Farrow, T., and Vedral, V., Scale-estimation of quantum coherent energy transport in multiple-minima systems, Scientific Reports 4, Article number: 5520.http://dx.doi.org/10.1038/srep05520, (2014).

[21]. Bochkov, G.N., and Kuzovlev, Y.E., General theory of thermal fluctuations in nonlinear systems, Zh. Eksp. Teor. Fiz. 72, 238, (1977).

[22]. Campisi, M., Hanggi, P. and Talkner, P., Colloquium: Quantum fluctuation relations: Foundations and applications, Reviews of Modern Physics, 83, 771, (2011).

[23]. Messiah, A., Quantum Mechanics (North-Holland,Amsterdam), (1962).

[24]. Wheeler, J.A., The'Past" and the Delayed Choice' Double-Slit experiment, in Lisa M. Dolling, Arthur F. Gianelli, Glenn N. Statilem, Readings in the Development of Physical Theory, 486ff, (1978)

[25]. Hellmuth, T., Walther, H., Zanjonc, A. and Schleich, W., Delayed-choice experiments in quantum interference. Physical Review A, 35, 2532, (1987).

[26]. Szabó, L., Quantum causal structures. Journal of mathematical physics, 27, 2709, (1986).

\section{APPENDIX}

Given:

$$
\begin{aligned}
& \phi\left(\frac{x+y}{2}\right)=a_{o} \exp \left(\frac{q}{2}(x+y)\right)+a_{o} \exp \left(-\frac{q}{2}(x+y)\right) \\
& \phi^{*}\left(\frac{x-y}{2}\right)=a_{o} \exp \left(\frac{q}{2}(x-y)\right)+a_{o} \exp \left(-\frac{q}{2}(x-y)\right)
\end{aligned}
$$

Therefore the composite function is as follows:

$$
\begin{aligned}
& \phi\left(\frac{x+y}{2}\right) \phi^{*}\left(\frac{x-y}{2}\right)=a_{o}^{2}\left(e^{q x}+e^{-q x}+e^{q y}+e^{q y}\right) \\
& \therefore W(x, \varepsilon)=a_{o}^{2} \int_{-\infty}^{+\infty}\left(e^{q x}+e^{-q x}+e^{q y}+e^{q y}\right) e^{-2 i \pi \varepsilon y} d y \\
& =a_{o}^{2} \int_{-\infty}^{+\infty}\left(e^{q x}+e^{-q x}\right) e^{-2 i \pi \varepsilon y} d y+a_{o}^{2} \int_{-\infty}^{+\infty}\left(e^{q y}+e^{-q y}\right) e^{-2 i \pi \varepsilon y} d y \\
& =a_{o} \cosh (q x) \delta(\varepsilon)+a_{o}^{2} \int_{-\infty}^{+\infty}\left(e^{q y}+e^{-q y}\right) e^{-2 i \pi \varepsilon y} d y
\end{aligned}
$$

The delta function could be defined as follows: 
$\delta(\varepsilon)=\left\{\begin{array}{lll}\infty & \text { if } & \varepsilon=0 \\ 0 & \text { if } & \varepsilon \neq 0\end{array}\right.$

Since $\varepsilon \neq 0$, therefore:

$\therefore W(x, \varepsilon)=a_{o}^{2} \int_{-\infty}^{+\infty}\left(e^{q y}+e^{-q y}\right) e^{-2 i \pi \varepsilon y} d y$

From equation (12),

$q^{2}=2 m\left(V_{o}-\eta-\hbar \omega\right) / \hbar^{2}$

Weak initial driving potential is set such that $V_{o} \approx \eta$. Therefore,

$q^{2}=(-2 m \omega) / \hbar$

$\therefore q=i \sqrt{2 m \omega / \hbar}$

Therefore Equation (A6) is solved by using the Fourier Transform definition:

$$
\begin{aligned}
W(x, \varepsilon) & =a_{o}^{2} \int_{-\infty}^{+\infty}\left(e^{q y}+e^{-q y}\right) e^{-2 i \pi \varepsilon y} d y \\
& =a_{o}^{2} \int_{-\infty}^{+\infty}\left(e^{q y}\right) e^{-2 i \pi \varepsilon y} d y+\int_{-\infty}^{+\infty}\left(e^{-q y}\right) e^{-2 i \pi \varepsilon y} d y \\
& =a_{o}^{2}\left[\delta\left(\varepsilon-\frac{\sqrt{2 m \omega / \hbar}}{2 \pi}\right)+\delta\left(\varepsilon+\frac{\sqrt{2 m \omega / \hbar}}{2 \pi}\right)\right]
\end{aligned}
$$

By the delta function property:

$$
\begin{aligned}
& \delta\left(u^{2}-v^{2}\right)=\frac{1}{2 v}\{\delta(u-v)+\delta(u+v)\} \quad: \quad v>0 \\
& W(x, \varepsilon)=a_{o}^{2} \delta\left(\varepsilon^{2}-\frac{m \omega}{\pi^{2} \hbar}\right)
\end{aligned}
$$

